\title{
Safety, efficacy, and patient acceptability of imiquimod for topical treatment of actinic keratoses
}

This article was published in the following Dove Press journal:

Clinical, Cosmetic and Investigational Dermatology

7 April 201 I

Number of times this article has been viewed

\section{Caroline Caperton \\ Brian Berman}

Department of Dermatology and Cutaneous Surgery, Miller School of Medicine, University of Miami, Miami, FL, USA
Correspondence: Brian Berman 1600 NW 10th Avenue, RSMB 2023A, Miami, FL 33।36, USA

Tel +l 3052435620

Email bberman@med.miami.edu
Abstract: Imiquimod, an immune-modulating imidazoquinoline compound, has been approved in topical formulation for the treatment of actinic keratoses, superficial basal cell carcinomas, and external genital warts. Its use in the treatment of field cancerization, in particular, has been rapidly evolving. With the recent approval of a new drug application for a new concentration, as well as generic formulations, this drug has emerged at the forefront of treatment for actinic keratoses, with improved dosage scheduling and more patients having access to generic options. In the nearly 15 years since its original approval by the Food and Drug Administration for the treatment of actinic keratoses in 1997, topical imiquimod has been reviewed and studied extensively, not only for its safety and efficacy, but also for its tolerability in patients. This paper provides an indepth review of the literature, and provides clinical evidence for its inclusion in the arsenal of treatment options for patients with actinic keratoses.

Keywords: imiquimod, actinic keratoses, field cancerization, field therapy

\section{Introduction}

Actinic keratoses are small, scaly, sandpaper-like papules found on sun-exposed areas of skin including the face, balding scalp, and arms. With an approximate four-year risk of evolving into basal cell carcinoma or squamous cell carcinoma of $1.6 \%$ and $2.6 \%$, respectively, these lesions are considered to be premalignant. ${ }^{1,2}$ There are many treatment options currently available for the treatment of actinic keratoses, including imiquimod, cryotherapy, 5-fluorouracil, 3\% diclofenac in 2.5\% hyaluronan gel, and topical photodynamic therapy, as well as new emerging potential therapies, such as ingenol-3-angelate. This review focuses on topical imiquimod and its history as a topical immune response modifier in the treatment of actinic keratoses.

Imiquimod, an imidazoquinoline compound, is a low molecular weight immune modifier which upregulates interferon-alpha, tumor necrosis factor-alpha, interleukin (IL)-1, IL-6, IL-8, IL-12, and other cytokines locally. It is capable of inducing both the innate and the adaptive immune responses in order to achieve enhancement of the endogenous immune response, and also upregulates the natural antitumor and antiviral cell response. In 1997, the Food and Drug Administration (FDA) approved topical imiquimod cream at a concentration of $5 \%$ to be used for the treatment of typical, nonhyperkeratotic, and nonhypertrophic actinic keratoses on the face or scalp in immunocompetent adults. In 2010, the FDA approved a 3.75\% imiquimod formulation for treatment of clinical typical, visible, or palpable actinic keratoses on the face or balding scalp. Formulations, indications, and the dosing regimen are listed in Table 1. As new formulations and treatment regimens are approved, more data 
Table I Imiquimod formulations, indications, and dosing regimens

\begin{tabular}{lll}
\hline Imiquimod formulation & Indication & Dosing regimen \\
\hline $5 \%$ cream, topical & $\begin{array}{l}\text { Treatment of clinically typical, nonhyperkeratotic, } \\
\text { nonhypertrophic actinic keratoses on the face or } \\
\text { scalp in immunocompetent adults; restricted to an area } \\
\text { of skin totaling } 25 \mathrm{~cm}^{2}\end{array}$ & 2 times per week for up to I6 weeks \\
& Treatment of clinically typical, visible, or & \\
$3.75 \%$ cream, topical & palpable actinic keratoses on the full face or & Daily use for 2 weeks, followed by 2 weeks of \\
& balding scalp & nontreatment, followed by 2 weeks of daily \\
use for a total of 6 weeks
\end{tabular}

will emerge on the safety and efficacy of topical imiquimod. Furthermore, we will gain more insight into its tolerability for patients.

\section{Overview of imiquimod Mechanism}

Structurally, the chemical nomenclature of imiquimod is 1-(2-methylpropyl)-1H-imidazo[4,5-c]quinolin-4-amine (Figure 1). ${ }^{3}$ Imiquimod acts as an immune modifier by targeting cytokine-producing cells, such as monocytes, macrophages, and dendritic cells bearing toll-like receptor $7 / 8 .{ }^{4}$ This first drug of its class acts via innate and acquired immune pathways in order to stimulate release of cytokines, such as tumor necrosis factor-alpha, IL-6, IL-12, and interferon-alpha, as well as activation of natural killer cells, antigen-presenting cells, $\mathrm{T}$ cells, and B cells (Figure 2). This cascade also results in the production of interferon-gamma by Th1 cells. ${ }^{5}$ Imiquimod induces cellular memory via the activation of effector T cells, Langerhans cells, lymphocytes, and macrophages, which circulate lymphatically and activate the adaptive immune response.

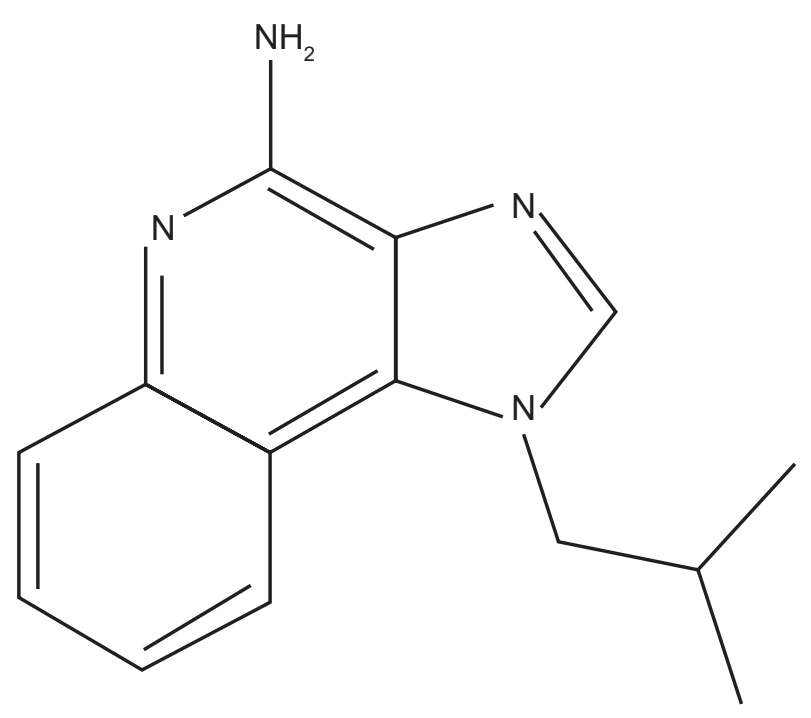

Figure I Chemical structure of Imiquimod.

\section{Metabolism and pharmacokinetics}

When applied topically, the half-life of imiquimod is approximately 30 hours. ${ }^{6}$ Systemic absorption of imiquimod is minimal when the cream is applied to intact skin, with $97 \%$ of the drug recovered from the dosing site in one study. Other studies have demonstrated no quantifiable metabolites in serum, with $<0.9 \%$ of a single $5 \mathrm{mg}$ dose excreted in urine and feces. ${ }^{7}$ There is no detectable radioactivity in serum. Kulp et al characterized serum markers in 19 subjects applying two packets $(18.75 \mathrm{mg}$ total) of imiquimod cream $3.75 \%$ once daily for 21 days to an area of skin on the face or balding scalp totaling approximately $200 \mathrm{~cm}^{2} .{ }^{8}$ After 21 days, the average maximum serum concentration was $0.323 \mathrm{mg} / \mathrm{mL}$, with a half-life of 29 hours. Researchers concluded that systemic exposure of imiquimod was low after 21 days of application.

Differences in the release rates of imiquimod between the generic and brand formulations have been studied in vitro. Due to differences in the compositions of the vehicle creams, there may be differences in the safety and efficacy of generic versus brand medications as well. To answer this question, a nationwide, multicenter, double-blind, 16-week study is underway to compare vehicles used in the generic and brand formulations of imiquimod cream 5\% for the treatment of actinic keratoses on the face or scalp. ${ }^{9}$

\section{Safety}

Due to the lack of data in animal models, the use of imiquimod is contraindicated during pregnancy. Imiquimod is listed as a Category $\mathrm{C}$ drug. Female patients of child-bearing age should be counseled regarding the importance of contraception before being prescribed imiquimod because its teratogenicity is as yet undetermined.

\section{Clinical data}

A double-blind, vehicle-controlled pilot study by Edwards el al evaluated imiquimod cream 5\% versus vehicle in 40 subjects with at least three actinic keratoses. ${ }^{10}$ When treated 


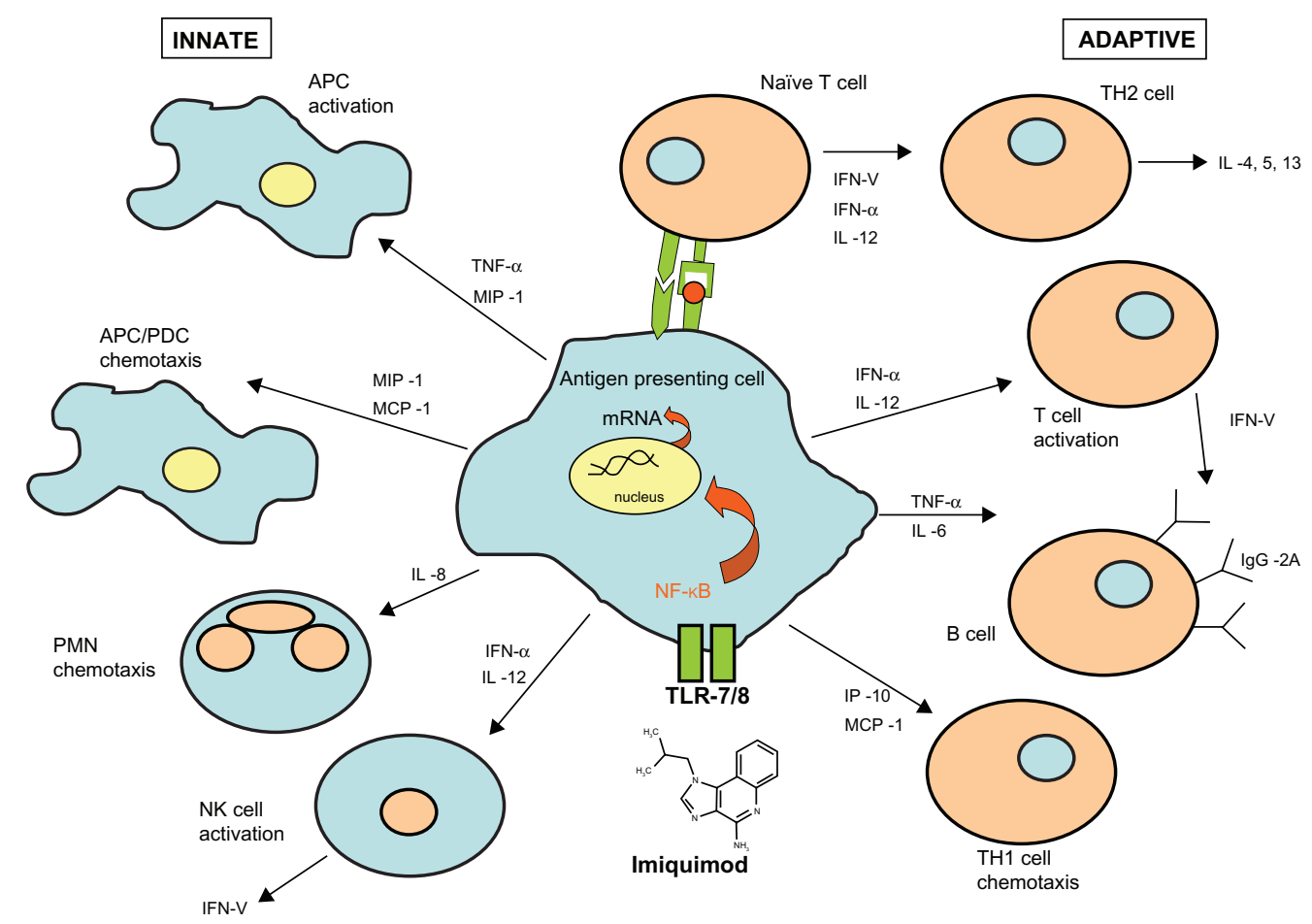

Figure 2 Mechanism of Imiquimod.

three times per week for up to 16 weeks, $75 \%$ of lesions cleared, compared with $12 \%$ in the vehicle group. Some of the first reported cases of imiquimod 5\% cream for the treatment of actinic keratoses were by Stockfleth et al in 2001. ${ }^{11}$ Six patients who had up to 10 actinic keratoses were treated 2-3 times per week for 6-8 weeks, with complete clearance and no recurrences one year later. Persaud et al conducted a study of 22 patients, each acting as their own control, applying topical imiquimod 5\% to one side of the body and vehicle to the other. ${ }^{12}$ At the end of eight weeks, a significant reduction in the number of lesions was observed for the imiquimod-treated side (from 10.1 to 6.2 ) compared with vehicle (8.1 to 7.6). An open-label trial of imiquimod 5\% cream was conducted by Salasche et al, involving 25 patients with 5-20 discrete actinic keratoses, treated three times per week for four weeks, followed by a rest period of four weeks. ${ }^{13}$ If any lesions remained, patients repeated the eight-week cycle up to a maximum of 24 weeks. Researchers noted that although $45.5 \%$ of sites were clear by the end of the first cycle, actinic keratoses continued to clear during the "therapeutic interval" rest period. Complete clearing was achieved in $82 \%$ of lesions, including subclinical lesions that were uncovered by treatment with imiquimod. This cycling approach was able to achieve high efficacy while minimizing adverse effects.

Two multicenter, randomized, double-blind, parallelgroup, vehicle-controlled Phase III trials were conducted to evaluate the efficacy of imiquimod 5\% cream for the treatment of actinic keratoses on the face and balding scalp. ${ }^{14}$ In total, 436 patients were randomized to apply either imiquimod $5 \%$ cream or vehicle once a day two times per week for 16 weeks. Complete clearance rates were $45.1 \%$ for the imiquimod group and $3.2 \%$ for the vehicle group. Although $17.7 \%$ of imiquimod-treated subjects reported severe erythema, only $3 \%$ discontinued the study due to adverse events. The second Phase III study involved application of imiquimod $5 \%$ or vehicle cream once a day three times per week for 16 weeks in 286 patients. ${ }^{15}$ Histology-proven complete clearance rates were observed in $57.1 \%$ of imiquimod-treated and $2.2 \%$ of vehicle-treated subjects, with dropout rates due to adverse events of $4 \%$ and $3 \%$, respectively.

Another pair of similar Phase III trials evaluated 492 patients randomized to apply either imiquimod 5\% cream or vehicle three times a week for 16 weeks. ${ }^{16}$ At eight weeks post-treatment, imiquimod-treated patients experienced an $86.6 \%$ reduction in the number of baseline lesions as compared with $14.3 \%$ for vehicle-treated patients. Long-term follow-up studies (1-2 years post-treatment) have shown continued clinical benefit of imiquimod treatment for actinic keratoses, with the majority of treated patients remaining clear, and low rates of recurrence. ${ }^{17}$

A meta-analysis comparing the efficacy of imiquimod $5 \%$ cream versus 5 -fluorouracil for the treatment of actinic 
keratoses on the face and scalp concluded that imiquimod was more efficacious than 5 -fluorouracil $(70 \% \pm 12 \%$ versus $52 \%$ $\pm 18 \%$, respectively). ${ }^{18}$ Tanghetti and Werschler conducted a trial assessing 39 patients randomized to receive either 5 -fluorouracil twice daily or imiquimod 5\% twice weekly for treatment of actinic keratoses for 16 weeks. ${ }^{19}$ At the end of the study at week 24 , reduction in the total actinic keratosis count was $94 \%$ for 5 -fluorouracil compared with $66 \%$ for imiquimod. However, more recent studies have reported sustained field clearance rates in all patients one year after treatment. In 75 patients treated with imiquimod 5\% three times weekly for four weeks, 5-fluorouracil twice daily for four weeks or cryotherapy (including also those not cleared at the end of therapy), sustained clearance was achieved in $73 \%, 33 \%$, and $4 \%$ of patients, respectively. ${ }^{20}$

Stockfleth et al evaluated the response to a shortened course of treatment. ${ }^{21}$ In an open-label Phase IIIb trial, 829 subjects applied imiquimod 5\% cream or vehicle to the treatment area three times weekly for four weeks, followed by a four-week period of no treatment. If actinic keratoses remained, then a second four-week course of treatment was initiated. Complete and partial ( $\geq 75 \%$ lesion reduction) clearance rates were $68.9 \%$ and $80.2 \%$, respectively, similar to those observed after 16-week treatment courses. To investigate whether a lower concentration of imiquimod might be efficacious in the treatment of actinic keratoses with reduced adverse reactions, two placebo-controlled studies were performed comparing imiquimod $2.5 \%$ and $3.75 \%$ to the face or balding scalp for two-week or three-week cycles. ${ }^{22,23}$ Results were similar for the two trials. In the two-week cycle study (daily treatment for two weeks, two weeks of no treatment, followed by another two weeks of daily treatment), mean reductions from baseline lesion count were $25 \%$ for placebo, $72 \%$ for imiquimod $2.5 \%$, and $82 \%$ for imiquimod $3.75 \%{ }^{22}$ Furthermore, the sustained clearance of lesions in these patients remained significant at one-year follow-up, with $33 \%$ of imiquimod $2.5 \%$ subjects and $41 \%$ of imiquimod $3.75 \%$ subjects remaining $100 \%$ clear of lesions.

When used in combination with cryotherapy, imiquimod has the ability to increase clearance of lesions. In a study of 63 subjects, when imiquimod 5\% cream was used post-cryotherapy, a significantly greater total number of actinic keratoses were cleared compared with vehicle (23\% versus 9\%). ${ }^{24}$ Imiquimod cream $3.75 \%$ was also evaluated post-cryotherapy in a multicenter, double-blind, vehicle-controlled study. ${ }^{25}$ Patients with at least five actinic keratoses on the face were treated with cryotherapy, leaving at least five lesions untreated. One to two weeks post-cryotherapy, imiquimod $3.75 \%$ or vehicle cream was applied to the treatment area in two-week cycles as described previously. Complete clearance rates were $30 \%$ for imiquimod versus 3\% for vehicle, with mean total lesion reductions of $87 \%$ versus $50 \%$, respectively.

To assess field-directed therapies used in combination, the sequential use of photodynamic therapy with aminolevulinic acid followed by imiquimod was performed in 25 subjects with facial actinic keratoses. ${ }^{26}$ One month after photodynamic therapy, imiquimod or vehicle was applied to the area twice weekly for 16 weeks. At one year of follow-up, median lesion reduction was $90 \%$ for imiquimod versus $75 \%$ for vehicle, with no subject discontinuing due to an adverse event.

Nonmelanoma skin cancer affects a disproportionately large number of immunosuppressed patients. Ulrich et al reported five of six organ transplant recipients being successfully treated for actinic keratoses with topical imiquimod 5\% cream. ${ }^{27}$ In this nonrandomized pilot study, imiquimod 5\% was applied 2-3 times per week for 12-16 weeks, with complete clearance of all lesions in five of six patients. Another study assessed graft function in 24 transplant patients receiving treatment with imiquimod, finding that it was not altered, and that therapy was well tolerated. ${ }^{28} \mathrm{~A}$ larger, multicenter, randomized, placebocontrolled study was undertaken by Ulrich et al involving 43 transplant patients with actinic keratoses. ${ }^{29}$ Patients applied imiquimod 5\% cream or vehicle three times per week for 16 weeks to a defined treatment area of $100 \mathrm{~cm} .^{2}$ Efficacy was similar to that in immunocompetent patients, with no patients experiencing transplant rejection or discontinuing due to drug-related adverse events.

\section{Patient acceptability}

Due to the ease, convenience, privacy, and autonomy associated with self-administration, treatment of dermatologic conditions with imiquimod cream is preferred by patients over physician-administered modalities. ${ }^{30}$ Patient preference should guide treatment, because clearance rates are significantly enhanced with good patient compliance with any treatment regimen.

Patient preferences comparing imiquimod 5\% and $3.75 \%$ have not been formally surveyed to date. However, the reduced total treatment time for the $3.75 \%$ cream from 16 weeks to six weeks offers a distinct advantage over the $5 \%$ cream in terms of patient compliance. Patients must still be made aware of the importance of prevention, sun avoidance, frequent skin examinations by a dermatologist, as well as the use of sun-protective outerwear and sun block. 


\section{Adverse effects}

The most common adverse effect from topically applied imiquimod cream is a localized skin reaction at the application site. Generally, imiquimod is well accepted, with few patients choosing to discontinue therapy or withdrawal from clinical trials due to intolerable side effects. Commonly, patients experience mild to moderate discomfort, with erythema, itching, burning, pain, edema, ulceration, and induration being reported most often. The erythema is characteristic of the local immune response, as imiquimod unmasks subclinical lesions and treats them in the process. A positive correlation exists between histologic clearance rates for actinic keratoses and the severity of erythema, crusting, scabbing, and erosion. ${ }^{31}$ Figure 3 provides an example of a characteristic patient response during a course of imiquimod therapy.

Rarely, imiquimod can trigger or exacerbate existing inflammatory conditions. Cases of arthritis,${ }^{32}$ pemphigus foliaceous, ${ }^{33}$ psoriasis, ${ }^{34}$ actinic cheilitis, ${ }^{35}$ pityriasis rubra pilaris,${ }^{36}$ alopecia, ${ }^{37}$ erosive pustular dermatosis of the scalp, ${ }^{38}$ and subacute cutaneous lupus erythematosus ${ }^{39}$ attributed to treatment with imiquimod have been alleged in the literature.

\section{Cost analysis}

A pharmacoeconomic analysis of the treatment of actinic keratosis found that although imiquimod is the initially more expensive of the medications approved by the FDA, when treating multiple lesions, it offers an advantage over cryotherapy or photodynamic therapy with 5-aminolevulinic acid in that patients do not need to return over multiple days for in-office treatment, thus reducing the cost to the patient. ${ }^{40}$

Studies have demonstrated a low incidence of new actinic keratotic lesions after treatment with imiquimod, due to its ability to induce immunologic memory. ${ }^{41}$ This long-term clearance offers an advantage in terms of the cost of retreating areas of recurrence. By treating the field of cancerization, subclinical lesions are eradicated, thus eliminating the need to wait until these lesions are clinically visible and further progressed.

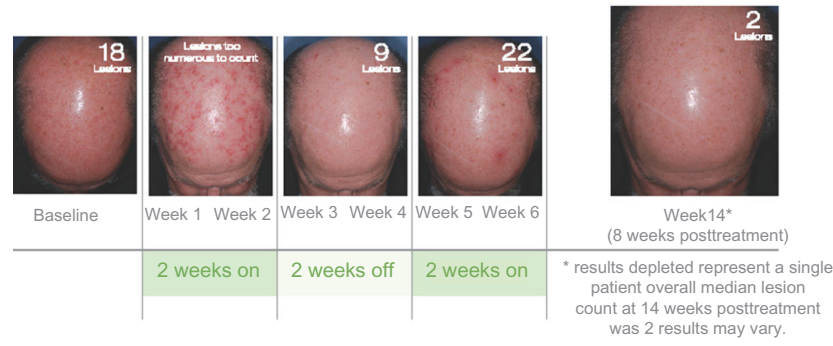

Figure 3 Characteristic patient response during a course of imiquimod therapy for actinic keratoses.

\section{Conclusion}

Topical imiquimod has proven to be safe and efficacious in the treatment of actinic keratoses, with tolerable transient local adverse effects. The ability of imiquimod to elicit an immune response and to treat subclinical lesions makes it among the most strategic therapies for these patients. With the availability of two concentrations, as well as generic formulations, imiquimod cream will no doubt continue to be used as effective monotherapy for patients with field cancerization in larger exposed skin areas. The research reviewed here highlights over a decade of clinical data on topical imiquimod. Field-directed therapies for the treatment of actinic keratoses will continue to emerge in this rapidly evolving field. Although no topical therapy has $100 \%$ efficacy without recurrence, imiquimod is among the forefront of these therapies, with much evidencebased justification for its place in the dermatologist's arsenal.

\section{Disclosure}

BB is a consultant for Graceway Pharmaceuticals, PharmaDerm, and CHS Pharma. CC has no conflicts of interest to report.

\section{References}

1. Grossman D, Leffell DJ. The molecular basis of nonmelanoma skin cancer. Arch Dermatol. 1997;133(10):1263-1270.

2. Criscione VD, Weinstock MA, Naylor MF, et al. Actinic keratoses: Natural history and risk of malignant transformation in the Veterans Affairs Topical Tretinoin Chemoprevention Trial. Cancer. 2009; 115(11):2523-2530.

3. Buck HW. Imiquimod (Aldara cream). Infect Dis Obstet Gynecol. 1998; 6(2):49-51.

4. Hemmi H, Kaisho T, Takeuchi O, et al. Small anti-viral compounds activate immune cells via the TLR7 MyD88-dependent signaling pathway. Nat Immunol. 2002;3(2):196-200.

5. Miller RL, Gerster JF, Owens ML, et al. Imiquimod applied topically: A novel immune response modifier and new class of drug. Int $J$ Immunopharmacol. 1999;21(11):1-14.

6. Owens ML, Bridson WE, Smith SL, Myers JA, Fox TL, Wells TM Percutaneous penetration of Aldara ${ }^{\mathrm{TM}}$ cream, $5 \%$ during the topical treatment of genital and perianal warts. Poster presented at the 46th annual clinical meeting of the American College of Obstetricians and Gynecologists, New Orleans, LA, May 9-13, 1998

7. Tygum KI, Smith SL, Myers JA, et al. Percutaneous penetration of $\left[{ }^{14} \mathrm{C}\right]$-imiquimod from a single application of cream. Pharm Res. 1995; 12(9 Suppl):Abstr 7339.

8. Kulp J, Levy S, Fein MC, Adams M, Furst J, Meng TC. Pharmacokinetics of imiquimod $3.75 \%$ cream applied daily for 3 weeks to actinic keratoses on the face and/or balding scalp. Arch Dermatol Res. 2010; 302(7):539-544

9. US Food and Drug Administration. Bioequivalence of generic imiquimod cream, 5\% when compared to Aldara ${ }^{\mathrm{TM}}$ (imiquimod) cream, $5 \%$ in the treatment of actinic keratosis. Clinicaltrials.gov Identifier: NCT00948428. Available at: http://clinicaltrials.gov/ct2/ show/NCT00948428. Accessed March 22, 2011.

10. Edwards L, Owens ML, Andres KL, et al. A pilot study evaluating imiquimod $5 \%$ cream versus vehicle in the treatment of actinic keratoses. Poster presented at the 58th annual meeting of the American Academy of Dermatology. San Francisco, CA, March 10-15, 2000. 
11. Stockfleth E, Meyer T, Benninghoff B, Christophers E. Successful treatment of actinic keratosis with imiquimod cream 5\%: A report of six cases. Br J Dermatol. 2001;144(5):1050-1053.

12. Persaud AN, Shamuelova E, Sherer D, et al. Clinical effect of imiquimod $5 \%$ cream in the treatment of actinic keratosis. J Am Acad Dermatol. 2002;47(4):553-556.

13. Salasche SJ, Levine N, Morrison L. Cycle therapy of actinic keratoses of the face and scalp with 5\% topical imiquimod cream: An open-label trial. J Am Acad Dermatol. 2002;47(4):571-577.

14. Lebwohl M, Dinehart S, Whiting D, et al. Imiquimod 5\% cream for the treatment of actinic keratosis: Results from two phase III, randomized, double-blind, parallel group, vehicle-controlled trials. J Am Acad Dermatol. 2004;50(5):714-721.

15. Szeimies RM, Gerritsen MJ, Gupta G, et al. Imiquimod 5\% cream for the treatment of actinic keratosis: Results from a phase III, randomized, double-blind, vehicle-controlled, clinical trial with histology. J Am Acad Dermatol. 2004;51(4):547-555.

16. Korman N, Moy R, Ling M, et al. Dosing with $5 \%$ imiquimod cream 3 times per week for the treatment of actinic keratosis: Results of two phase 3, randomized, double-blind, parallel-group, vehicle-controlled trials. Arch Dermatol. 2005;141(4):467-473.

17. Lee PK, Harwell WB, Loven KH, et al. Long-term clinical outcomes following treatment of actinic keratosis with imiquimod $5 \%$ cream. Dermatol Surg. 2005;31(6):659-664.

18. Gupta AK, Davey V, McPhail H. Evaluation of the effectiveness of imiquimod and 5-fluorouracil for the treatment of actinic keratosis: Critical review and meta-analysis of efficacy studies. J Cutan Med Surg. 2005;9(5):209-214.

19. Tanghetti E, Werschler P. Comparison of 5\% 5-fluorouracil cream and $5 \%$ imiquimod cream in the management of actinic keratoses on the face and scalp. J Drugs Dermatol. 2007;6(2):144-147.

20. Krawtchenko N, Roewert-Huber J, Ulrich M, Mann I, Sterry W, Stockfleth E. A randomised study of topical 5\% imiquimod vs topical 5 -fluorouracil vs cryosurgery in immunocompetent patients with actinic keratoses: A comparison of clinical and histological outcomes including 1-year follow-up. Br J Dermatol. 2007;157 Suppl 2:34-40.

21. Stockfleth E, Sterry W, Carey-Yard M, Bichel J. Multicentre, openlabel study using imiquimod $5 \%$ cream in one or two 4-week courses of treatment for multiple actinic keratoses on the head. Br J Dermatol. 2007;157 Suppl 2:41-46.

22. Swanson N, Abramovits W, Berman B, Kulp J, Rigel DS, Levy S. Imiquimod $2.5 \%$ and $3.75 \%$ for the treatment of actinic keratoses: Results of two placebo-controlled studies of daily application to the face and balding scalp for two 2-week cycles. $J$ Am Acad Dermatol. 2010;62(4):582-590.

23. Hanke CW, Beer KR, Stockfleth E, Wu J, Rosen T, Levy S. Imiquimod $2.5 \%$ and $3.75 \%$ for the treatment of actinic keratoses: Results of two placebo-controlled studies of daily application to the face and balding scalp for two 3-week cycles. J Am Acad Dermatol. 2010;62(4): 573-581.

24. Tan JK, Thomas DR, Poulin Y, Maddin F, Tang J. Efficacy of imiquimod as an adjunct to cryotherapy for actinic keratoses. J Cutan Med Surg. 2007;11(6):195-201.
25. Jorizzo JL, Markowitz O, Lebwohl MG, et al. A randomized, doubleblinded, placebo-controlled, multicenter, efficacy and safety study of $3.75 \%$ imiquimod cream following cryosurgery for the treatment of actinic keratoses. J Drugs Dermatol. 2010;9(9):1101-1108.

26. Shaffelburg M. Treatment of actinic keratoses with sequential use of photodynamic therapy and imiquimod 5\% cream. J Drugs Dermatol. 2009;8(1):35-39.

27. Ulrich C, Busch JO, Meyer T, et al. Successful treatment of multiple actinic keratoses in organ transplant patients with topical 5\% imiquimod: A report of six cases. Br J Dermatol. 2006;155(2):451-454.

28. Ben M'barek L, Mebazaa A, Euvrard S, et al. $5 \%$ topical imiquimod tolerance in transplant recipients. Dermatology. 2007;215(2): 130-133.

29. Ulrich C, Bichel J, Euvrard S, et al. Topical immunomodulation under systemic immunosuppression: Results of a multicentre, randomized, placebo-controlled safety and efficacy study of imiquimod $5 \%$ cream for the treatment of actinic keratoses in kidney, heart, and liver transplant patients. Br J Dermatol. 2007;157 Suppl 2:25-31.

30. O'Mahoney C, Law C, Gollnick HPM, Marini M. New patient-applied therapy for anogenital warts is rated favourably by patients. Int $J$ STD AIDS. 2001;12:565-570.

31. Skinner R. Role of topical therapies in the management of cutaneous disease. J Cutan Med Surg. 2004;8 Suppl 3:22-31.

32. Benson E. Imiquimod: Potential risk of an immunostimulant. Australas J Dermatol. 2004;45(2):123-124.

33. Lin R, Ladd DJ, Powell DJ, Way BV. Localized pemphigus foliaceous induced by topical imiquimod. Arch Dermatol. 2004;140:889-890.

34. Fanti PA, Dika E, Vaccari S, Miscial C, Varotti C. Generalized psoriasis induced by topical treatment of actinic keratosis with imiquimod. Int J Dermatol. 2006;45(12):1464-1465.

35. Greenberg HL, Cohen JL, Rosen T, Orengo I. Severe reaction to 5\% imiquimod cream with excellent clinical and cosmetic outcomes. J Drugs Dermatol. 2007;6(4):452-458.

36. Yang FC, Jessup C, Dahiya M, Reynolds R. Pityriasis rubra pilaris exacerbation with topical use of imiquimod. Int J Dermatol. 2008; 47(10):1076-1078.

37. Conde J, Davis K, Ntuen E, Balmer N, Jones D, McMichael A. A case of imiquimod-induced alopecia. J Dermatolog Treat. 2010;21(2): 122-124.

38. Vaccaro M, Barbuzza O, Guarneri B. Erosive pustular dermatosis of the scalp following treatment with topical imiquimod for actinic keratosis. Arch Dermatol. 2009;145(11):1340-1341.

39. Burnett TJ, English JC 3rd, Ferris LK. Development of subacute cutaneous lupus erythematosus associated with the use of imiquimod to treat actinic keratoses. J Drugs Dermatol. 2010;9(8):1022-1024.

40. Gold MH. Pharmacoeconomic analysis of the treatment of multiple actinic keratoses. J Drugs Dermatol. 2008;7(1):23-25.

41. Stockfleth E, Christophers E, Benninghoff B, Sterry W. Low incidence of new actinic keratoses after topical $5 \%$ imiquimod cream treatment: A long-term follow-up study. Arch Dermatol. 2004;140:1542.
Clinical, Cosmetic and Investigational Dermatology

\section{Publish your work in this journal}

Clinical, Cosmetic and Investigational Dermatology is an international, peer-reviewed, open access, online journal that focuses on the latest clinical and experimental research in all aspects of skin disease and cosmetic interventions. All areas of dermatology will be covered; contributions will be welcomed from all clinicians and

\section{Dovepress}

basic science researchers globally. This journal is indexed on CAS The manuscript management system is completely online and includes a very quick and fair peer-review system, which is all easy to use. Visit http://www.dovepress.com/testimonials.php to read real quotes from published authors. 